\title{
Metastatic extraskeletal Ewing's sarcoma treated with trabectedin: A case report
}

\author{
JORGE HERNANDO-CUBERO, PILAR SANZ-MONCASI, ALBA HERNÁNDEZ-GARCÍA, \\ ISABEL PAJARES-BERNARD and JAVIER MARTÍNEZ-TRUFERO
}

\author{
Department of Medical Oncology and Pathology, University Hospital Miguel Servet, 50008 Zaragoza, Spain
}

Received February 5, 2016; Accepted June 27, 2016

DOI: $10.3892 / \mathrm{ol} .2016 .4950$

\begin{abstract}
The Ewing's sarcoma family of tumors (ESFT) comprises a number of rare malignant tumors. Standard first-line treatment for patients with these tumors includes chemotherapy with a five-drug regimen of vincristine, doxorubicin (Adriamycin ${ }^{\circledR}$ ) and cyclophosphamide, alternating with ifosfamide and etoposide (VAC/IE). In cases of inadequate response, there are a number of second-line regimens available. However, further treatment options are required for those patients with disease unresponsive to standard treatment. Trabectedin is a novel treatment option for patients with ESFT. The present study reports the case of a Caucasian 69-year-old female patient who presented with a soft tissue mass on the chest wall that had developed 7 months earlier. A computed tomography scan revealed a $9 \times 8 \times 7-\mathrm{cm}$ mass on the anterior chest wall above the pectoral muscle. Histopathological evaluations and molecular analysis indicated that it was consistent with a metastatic extraskeletal Ewing's sarcoma. The patient was treated with an alternating VAC/IE regimen; however, an inadequate response was observed. The patient received second-line treatment with a gemcitabine and dacarbazine combination regimen, but the disease progressed. Subsequently, treatment with trabectedin $\left(1.5 \mathrm{mg} / \mathrm{m}^{2}\right.$ as a $24-\mathrm{h}$ continuous infusion every 3 weeks) was initiated. Trabectedin treatment resulted in long-lasting (18 months) progression-free survival. It is vital that novel drugs continue to being developed for patients with ESFT following progression
\end{abstract}

Correspondence to: Dr Jorge Hernando-Cubero, Department of Medical Oncology and Pathology, University Hospital Miguel Servet, Paseo Isabel la Católica 1-3, 50008 Zaragoza, Spain

E-mail: hernandocubero@hotmail.es

Abbreviations: CT, computed tomography; ESFT, Ewing's sarcoma family of tumors; EWS, Ewing's sarcoma; FLI1, friend leukemia integration $1 \mathrm{OS}$, overall survival; PFS, progression-free survival; PNET, primitive neuroectodermal tumors; TRSs, translocation-related sarcomas; VAC/IE, vincristine, doxorubicin (Adriamycin $^{\circledR}$ ) plus cyclophosphamide alternating with ifosfamide and etoposide

Key words: extraskeletal metastases, Ewing's sarcoma, metastatic sarcoma, palliative chemotherapy, trabectedin subsequent to standard chemotherapy. The current report presents a case of a patient with metastatic, pre-treated Ewing's sarcoma achieving disease stabilization with trabectedin. Based on these results and the observed tolerability profile, trabectedin represents an alternative treatment for patients with ESFT. Further studies are required in order to determine the efficacy of trabectedin as monotherapy or in combination with other drugs. It is also important to identify which tumor subtypes, specific translocations and patient profiles will benefit the most from treatment with trabectedin.

\section{Introduction}

The Ewing's sarcoma family of tumors (ESFT) consists of a group of neoplasms with a number of characteristics in common, including the presence of small round cells, a shared neuroectodermal origin, an immunohistochemical panel positive for cluster of differentiation (CD)99 and the Ewing's sarcoma (EWS)-friend leukemia integration 1 (FLI1) transcription factor translocation $\mathrm{t}(11 ; 22)(\mathrm{q} 24 ; \mathrm{q} 12)$, which occurs in $85 \%$ of cases $(1,2)$. The EWS-E26 transformation-specific (ETS)-related gene (ERG) translocation $\mathrm{t}(21 ; 22)(\mathrm{q} 21 ; \mathrm{q} 22)$ is present in $5-10 \%$ of cases (2). Other rarer translocations described include the genes ETS variant (ETV)1, ETV4 or fifth Ewing's sarcoma variant (FEV), forming $\mathrm{t}(7 ; 22)(\mathrm{p} 22 ; \mathrm{q} 12), \mathrm{t}(17 ; 22)(\mathrm{q} 12 ; \mathrm{q} 12)$ or $\mathrm{t}(2 ; 22)(\mathrm{q} 35 ; \mathrm{q} 12)$, respectively $(2,3)$. Certain cases have translocations that involve the fused in sarcoma (FUS) gene, such as FUS-ERG $\mathrm{t}(16 ; 21)$ (p11;q24) or FUS-FEV t(2;16)(q35;p11) (2).

Several tumors that had traditionally been considered separate entities are now included in this family of tumors, including skeletal Ewing's sarcoma, extraskeletal Ewing's sarcoma, primitive neuroectodermal tumors (PNETs) and Askin's tumor $(1,2)$. This classification allows rare tumors such as PNETs to benefit from chemotherapy regimens traditionally reserved for skeletal Ewing's sarcoma $(1,2)$. Chemotherapy for these patients is based on the vincristine, doxorubicin (Adriamycin ${ }^{\circledR}$ )and cyclophosphamide alternating with ifosfamide and etoposide (VAC/IE) regimen (4). Regimens used in subsequent lines include cyclophosphamide/topotecan, irinotecan/temozolomide and docetaxel/gemcitabine $(1,2)$. However, since patients with sarcomas are typically young and achieve good clinical status with subsequent lines of chemotherapy $(1,2)$, alternate therapies must be identified if the disease progresses following standard chemotherapy. The current report presents a 
case of a patient with metastatic extraskeletal Ewing's sarcoma who responded well to trabectedin.

\section{Case report}

A Caucasian 69-year-old female patient with a history of high blood pressure, diabetes and dyslipidemia presented to the University Hospital Miguel Servet (Zaragoza, Spain) in June 2011 for diagnosis and treatment of a suspicious soft tissue mass on the chest wall. The patient neither smoked, had any other toxic habits or had a family history of neoplasms. The patient had previously consulted her primary care doctor 7 months earlier (in November 2010) regarding a soft tissue mass on the chest wall; however, no scans were performed at that time. Later, in June 2011, during a pre-operative evaluation for knee surgery, multiple lung nodules were observed on chest X-ray; thus, a full investigation was conducted.

The computed tomography (CT) scan revealed a 9x8x7-cm mass on the anterior chest wall above the pectoral muscle, two enlarged internal mammary lymph nodes measuring 12 and $16 \mathrm{~mm}$, respectively, a right axillary lymphadenopathy (14-mm) and multiple bilateral lung metastases (Fig. 1). The biopsy of the superficial mass was consistent with a PNET/extraskeletal Ewing's sarcoma. Immunohistochemical staining demonstrated that the tumor cells were positive for CD99 (anti-CD99; dilution, 1:20-1:200; catalog number 23079-1-AP; ProteinTech Group, Inc., Chicago, IL, USA) and vimentin (anti-vimentin; dilution 1:1,000; catalog number 18-0052; Invitrogen; Thermo Fisher Scientific, Inc., Waltham, MA, USA). Proliferative activity, detected by the Ki-67 index, was estimated to be $20 \%$. A molecular analysis was performed by fluorescence in situ hybridization using a commercially available Vysis LSI EWSR1 (22q12) Dual Colour, Break Apart Rearrangement Probe (Abbott Molecular Inc., Des Plaines, IL, USA), which revealed that $8 \%$ of the tumor cells were positive for the EWS-RNA-binding protein 1 translocation. The patient was then referred to the Department of Oncology at the University Hospital Miguel Servet for assessment. The patient was diagnosed with metastatic extraskeletal Ewing's sarcoma. Chemotherapy was initiated with an alternating VAC/IE regimen (vincristine, $2 \mathrm{mg} / \mathrm{m}^{2}$, Adriamycin ${ }^{\circledR}$, $75 \mathrm{mg} / \mathrm{m}^{2}$ and cyclophosphamide, $1,200 \mathrm{mg} / \mathrm{m}^{2}$, alternating with ifosfamide, $1,800 \mathrm{mg} / \mathrm{m}^{2}$ and etoposide, $100 \mathrm{mg} / \mathrm{m}^{2}$ ).

Following four VAC/IE cycles, in November 2011, stable disease in the chest wall mass and lungs was observed on CT scan of the chest. Given this poor response to the VAC/IE regimen and the patient's advanced age, it was decided to administrate palliative chemotherapy with a combination of gemcitabine $\left(1,800 \mathrm{mg} / \mathrm{m}^{2}\right)$ and dacarbazine $\left(500 \mathrm{mg} / \mathrm{m}^{2}\right)$ every 2 weeks. However, in February 2012, following 8 cycles of treatment, a CT scan revealed that the lung cancer had progressed in spite of therapy (Fig. 2).

In March 2012, treatment with trabectedin $\left(1.5 \mathrm{mg} / \mathrm{m}^{2}\right.$ as a 24-h continuous infusion every 3 weeks) was initiated. The patient received a total of 23 cycles between March 2012 and August 2013, with all radiological examinations revealing stable disease of the chest wall mass and the lung metastases (Fig. 3). In terms of toxicity, the patient exhibited only grade 1 asthenia.

From mid 2013, the patient experienced severe lower back pain and was diagnosed with severe spondyloarthropathy at L5-S1 with S1 compression of degenerative origin, which was

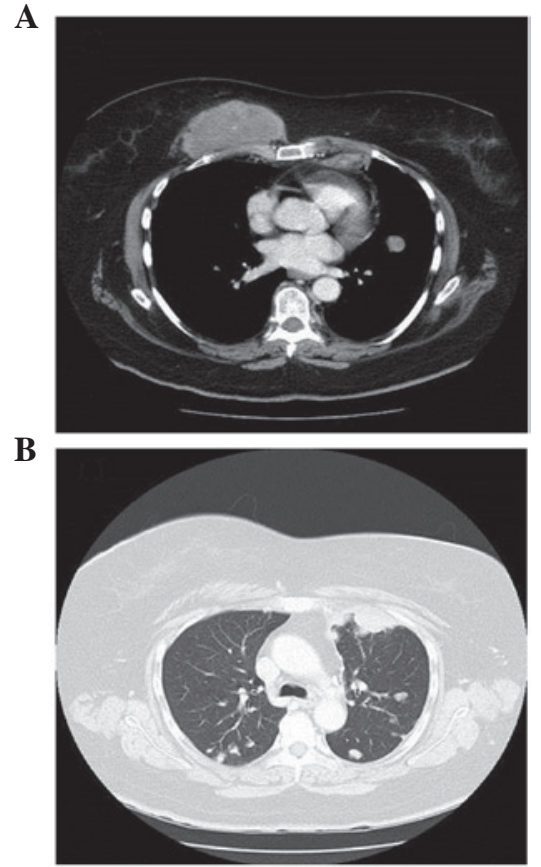

Figure 1. (A and B) Thorax computed tomography scans of the patient (November 2011) revealing (A) a chest wall mass with (B) bilateral lung metastases

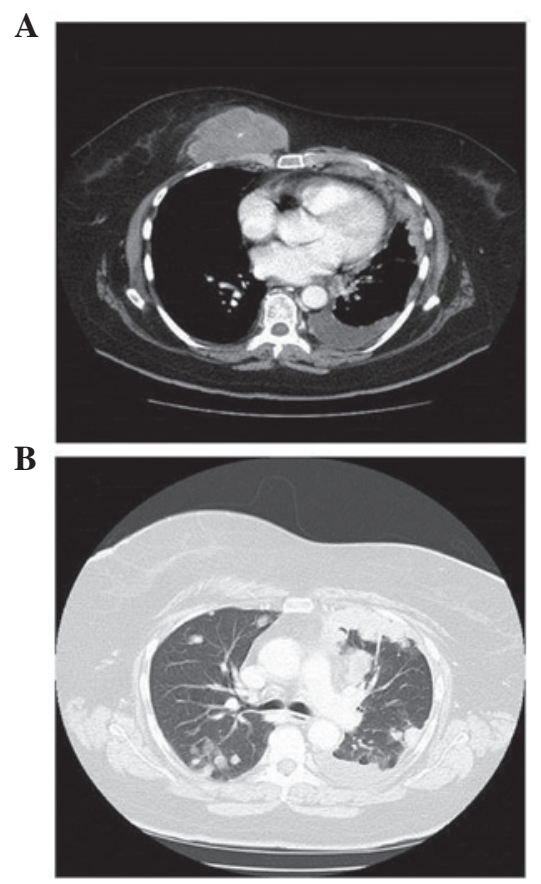

Figure 2. (A and B) Repeat thorax computed tomography scans 3 months after the initial scan (February 2012) revealing progressive disease following 8 cycles of gemcitabine and dacarbazine in the (A) chest wall mass and (B) bilateral lung metastases.

unrelated to the sarcoma. The Department of Trauma at the University Hospital Miguel Servet did not consider the patient eligible for surgery due to her history of cancer. Despite the use of the three-step analgesic ladder for the management of pain with escalating opioid dosages, the patient's condition progressively worsened with serious functional limitation and 
A
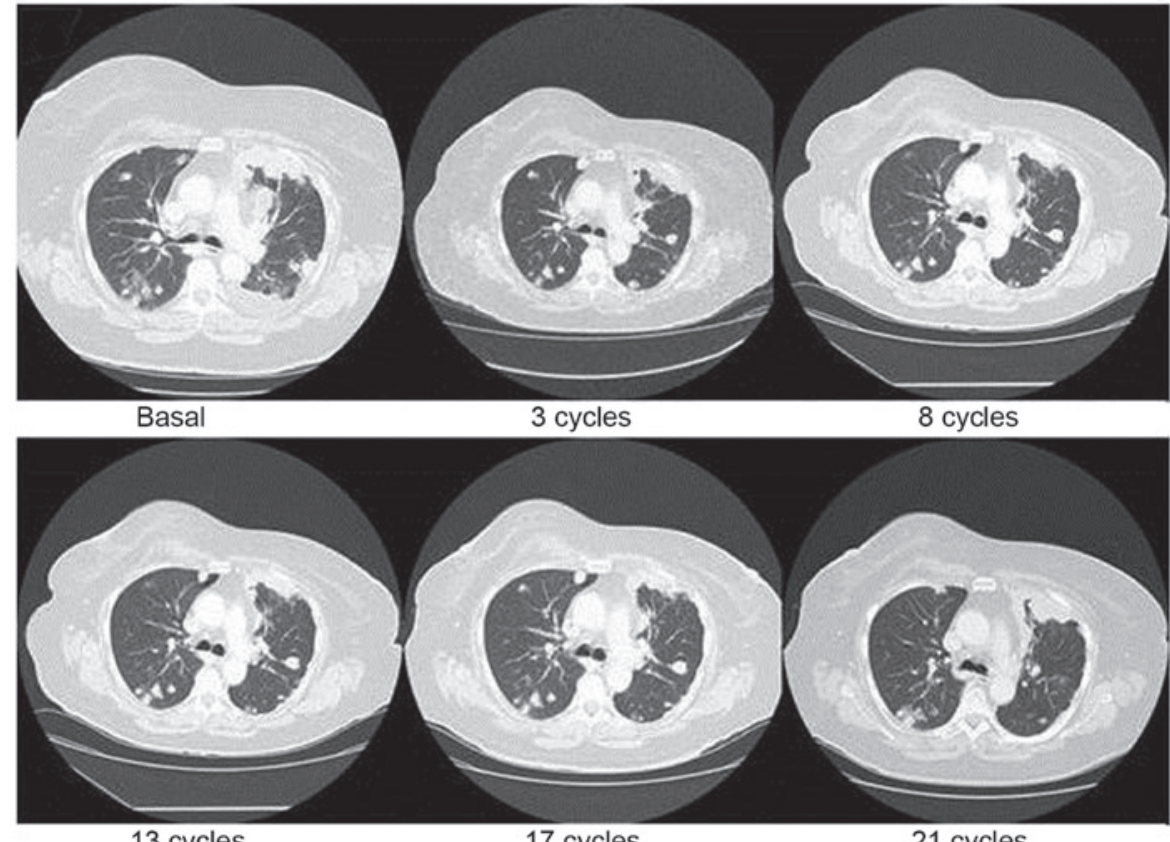

B

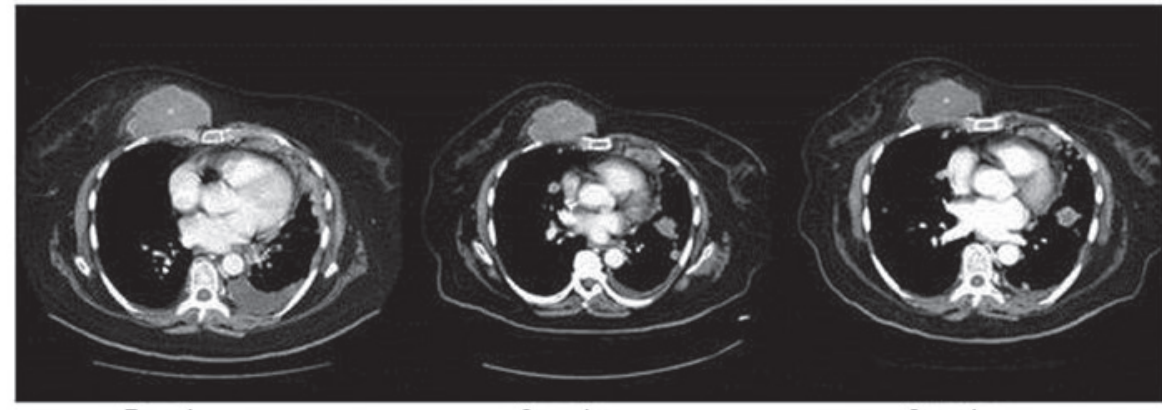

Basal

3 cycles

8 cycles

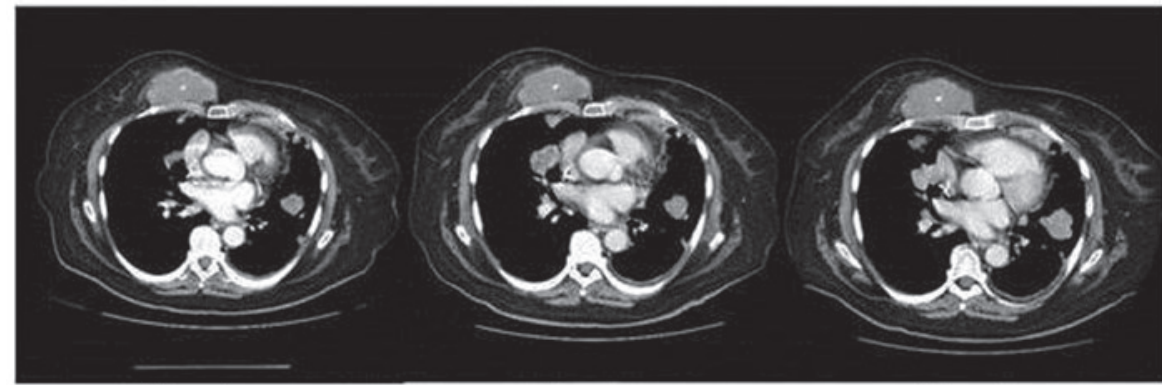

13 cycles

17 cycles

21 cycles

Figure 3. (A and B) Sequential thorax computed tomography scans from March 2012 to August 2013 revealing prolonged disease stabilization in the (A) bilateral lung metastases and (B) chest wall mass.

confinement to bed due to her lower back pain. In September 2013, the patient was admitted to hospital, contracted hospital-acquired pneumonia and succumbed to the disease. Imaging tests performed during this admission revealed no signs of tumor progression. Therefore, the patient had stable disease, both clinically and radiographically, for 18 months from the commencement of treatment with trabectedin.

\section{Discussion}

Ewing's sarcoma belongs to a diverse group of small-round-cell tumors characterized by the same chromosomal translocation, $\mathrm{t}(11 ; 22)$ (1). ESFT most commonly affects children, adolescents and young adults (1). There are no treatment guidelines for older patients with Ewing's sarcoma. Based in our own experience with older patients, it may be very difficult to maintain the full dosages of the drugs that the most aggressive chemotherapy regimens recommend in this population, as elderly patients may be at higher risk of toxicity than the young ones due to factors unrelated to treatment.

Following a poor response to the traditional first-line treatment regimen of alternating VAC/IE, the present patient was administered combination therapy with gemcitabine and dacarbazine every 2 weeks. Studies in paediatric populations 
Table I. Phase I and II clinical trials of T in Ewing's sarcoma/PNET.

\begin{tabular}{|c|c|c|c|c|c|c|c|c|}
\hline $\begin{array}{l}\text { First } \\
\text { author (ref.) }\end{array}$ & Year & Phase & $\begin{array}{c}\text { Dose } \\
\text { (infusion duration) }\end{array}$ & $\begin{array}{l}\text { Patients with Ewing's } \\
\text { sarcoma/PNET, n }\end{array}$ & CR & PR & SD & PD \\
\hline Lau (20) & 2005 & I & $\mathrm{T}: 1,100-1,300 \mathrm{ng} / \mathrm{m}^{2}(3 \mathrm{~h}) \mathrm{q} 3 \mathrm{w}$ & 3 (children) & 1 & 0 & 1 & 1 \\
\hline Papadopoulos (21) & 2006 & I & Paclitaxel + T: $525-775 \mathrm{ng} / \mathrm{m}^{2}(3 \mathrm{~h}) \mathrm{q} 2 \mathrm{w}$ & 1 (adult) & 1 & 0 & 0 & 0 \\
\hline Dileo (22) & 2007 & I & Cisplatin + T: 0.6 mg $/ \mathrm{m}^{2}(3 \mathrm{~h}) \mathrm{q} 3 \mathrm{w}$ & 29 (adults) & 0 & 6 & 4 & 19 \\
\hline Baruchel (23) & 2012 & II & $\mathrm{T}: 1.5 \mathrm{mg} / \mathrm{m}^{2}(24 \mathrm{~h}) \mathrm{q} 3 \mathrm{w}$ & 8 (children) & 0 & 0 & 1 & 7 \\
\hline
\end{tabular}

CR, complete response; PD, progressive disease; PNET, primitive neuroectodermal tumor; PR, partial response; qnw, every $\mathrm{n}$ weeks; $\mathrm{SD}$, stable disease; $\mathrm{T}$, trabectedin.

have demonstrated that combination therapy with gemcitabine and docetaxel exerts antitumor activity against advanced sarcoma (primarily Ewing's sarcoma) while maintaining a good quality of life $(5,6)$. Therefore, this combination is usually recommended as the second-line treatment for patients with Ewing's sarcoma; however, this regimen was ruled out in the present case due to the patient's age and health status (7). Instead, a combination of gemcitabine and dacarbazine appeared to be a suitable regimen for the patient, based on the palliative approach undertaken and her advanced age.

The efficacy of the gemcitabine and dacarbazine regimen in soft tissue sarcomas was assessed in a phase II clinical trial by García-Del-Muro et al (8) The trial compared the combination of gemcitabine $\left(180 \mathrm{mg} / \mathrm{m}^{2}\right)$ followed by dacarbazine $\left(500 \mathrm{mg} / \mathrm{m}^{2}\right)$ every 2 weeks with dacarbazine alone $\left(1,200 \mathrm{mg} / \mathrm{m}^{2}\right)$ every 3 weeks. The combination regimen demonstrated a greater progression-free survival (PFS) rate than dacarbazine alone, with a similar toxicity profile (8). However, in the present case, the patient did not benefit from this treatment regimen, as lung progression occurred following 8 cycles of treatment. It was then decided to commence treatment with trabectedin, with dosages administered according to the Summary of Product Characteristics (www.ema.europa. eu/docs/en_GB/document_library/EPAR_-_Product_Information/human/000773/WC500045832.pdf).

Trabectedin (also known as Ecteinascidin 743) is a drug of marine origin obtained from Ecteinascidia turbinata (9). By binding to the minor groove of DNA, trabectedin can induce damage by altering the normal function of DNA repair and transcription processes, thus inhibiting cell proliferation and differentiation and leading to cell death (9). This drug has demonstrated efficacy against soft tissue sarcomas in patients pre-treated with standard chemotherapy in several phase II trials (10-13). Based on the results of these studies, trabectedin was approved by the European Medicines Agency (London, UK) for the treatment of adult patients with advanced-stage soft tissue sarcomas in whom treatment with anthracyclines and ifosfamide has failed, or for those who are not candidates for these therapies (10-13). Although the efficacy is based on findings mainly for liposarcoma and leiomyosarcoma patients and does not include patients with Ewing's sarcoma or PNET, its approval has been extended to other subtypes of sarcoma (10-13), which enables the use of trabectedin as a treatment in other rare cancer histologies.
Trabectedin has also exhibited efficacy in translocationrelated sarcomas (TRSs), including synovial sarcoma, alveolar soft part sarcoma and endometrial stromal sarcoma (14-17). A recent phase II trial in a Japanese population evaluated the efficacy and safety of trabectedin compared with second-line or later supportive treatment in TRS (18). A total of 76 patients with extraskeletal Ewing's sarcoma, myxoid liposarcoma or synovial sarcoma were randomized. The analysis of the results revealed higher rates of PFS and overall survival (OS) that were statistically significant in favour of treatment with trabectedin, both in the overall population (median PFS, 5.6 vs. 0.9 months; hazard ratio, $0.07 ; \mathrm{P}<0.0001$ ) and in the analysis by subtype (18).

There are limited reported data regarding the efficacy of trabectedin in the treatment of extraskeletal Ewing's sarcoma/PNET. It has been demonstrated in vitro that trabectedin is active against cell lines that express the EWS-FLI1 translocation (19). A number of phase I clinical trials have reported promising results (20-22), even achieving complete responses in 2 cases [10 months in the study by Lau et al (20) and 16 months in the study by Chu et al (21)]. In spite of these findings, a phase II clinical trial reported that trabectedin conferred no treatment benefit in this tumor type (Table I) (23).

Recently, Grohar et al (24) highlighted that Ewing's sarcoma is sensitive to trabectedin, and suggested that the drug's apparent lack of clinical efficacy in this subtype of sarcoma could be due to its pharmacokinetics (24). In a phase II study (23), the level of trabectedin in the blood was $3.26 \pm 2.95 \mathrm{nmol} / \mathrm{l}$, which was lower than the 5-10 nmol/l level that had demonstrated in vitro activity (24). By contrast, in the phase I study by Lau et al (20), the levels of trabectedin reached $6.02 \pm 3.28 \mathrm{ng} / \mathrm{ml}$ in patients on $1,100 \mathrm{ng} / \mathrm{m}^{2}$ trabectedin and $10.52 \pm 5.00 \mathrm{ng} / \mathrm{ml}$ in patients on $1,300 \mathrm{ng} / \mathrm{m}^{2}$ trabectedin.

The study by Grohar et al (24) also analyzed the effect of combining trabectedin with SN38, an active metabolite of irinotecan. In this in vitro model, trabectedin blocked EWS-FLI1 and secondarily suppressed the production of the Werner syndrome adenosine triphosphate-dependent helicase protein, which is important in telomere maintenance, thereby sensitizing the cell to the toxic effect that camptothecin derivatives such as irinotecan exert on DNA. Thus, the combination of trabectedin and SN38 had a synergistic effect, requiring a lower concentration of trabectedin to have an antitumor effect.

In 2013, Cesne et al (25) published a review of five phase II studies in which 350 patients with soft tissue sarcoma were treated with trabectedin. The review evaluated the effect of age 
on the efficacy and safety of trabectedin. Patients were stratified into one cohort under 60 years of age $(n=267)$ and another over 60 years of age $(n=83)$. The retrospective analysis revealed no significant differences in PFS or OS between the two age groups, and no evidence of cumulative toxicity was observed. Trabectedin has an acceptable and manageable safety profile, and its antitumor activity in elderly patients with soft tissue sarcoma is similar to that observed in the general population (25). Based on these findings, trabectedin was recommended as part of palliative care for the patient described in the present case report.

The current case report highlights the clinical outcome of a patient with extraskeletal Ewing's sarcoma treated with trabectedin who achieved a PFS longer than any PFS reported thus far in the literature, following progression with first- and second-line chemotherapy. This is one of the few cases demonstrating that trabectedin has a clinical benefit in this type of tumor, and also confirms its excellent tolerability profile. The PFS could have been even longer upon treatment with trabectedin, since the patient succumbed to causes unrelated to the tumor. It should be emphasized that the patient received treatment as a 24-h continuous infusion, and although this regimen did not demonstrate efficacy in the phase II clinical trial by Baruchel et al (23), it can be hypothesized that this dosing regimen may lead to therapeutic levels in the tumor, as has been observed in vitro (23).

The authors consider that it is important to continue developing novel drugs for patients with ESFT following progression upon standard chemotherapy. Trabectedin may represent an alternative for patients with these tumors, although further studies are required in order to determine the efficacy of the treatment as monotherapy and combined with other drugs, including irinotecan, based on the potential synergy of these two drugs. It may also be important to identify which tumor subtypes, specific translocations and patient profiles will benefit the most from trabectedin.

In summary, the current report presents a case of a patient with metastatic, pre-treated Ewing's sarcoma achieving disease stabilization with trabectedin. Based on these findings and the observed tolerability profile, trabectedin represents an alternative treatment for patients with ESFT.

\section{Acknowledgements}

The authors would like to thank Miss Therese Chapman, who provided English editing and styling for submission on behalf of Springer Healthcare Ltd. (Chester, UK). This medical writing assistance was funded by PharmaMar S.A. (Madrid, Spain).

\section{References}

1. Delattre O,Zucman J, Melot T, Garau XS, Zucker JM, Lenoir GM, Ambros PF, Sheer D, Turc-Carel C, Triche TJ, et al: The Ewing family of tumors-a subgroup of small-round-cell tumors defined by specific chimeric transcripts. N Engl J Med 331: 294-299, 1994.

2. Ng TL, O'Sullivan MJ, Pallen CJ, Hayes M, Clarkson PW, Winstanley M, Sorensen PH, Nielsen TO and Horsman DE: Ewing sarcoma with novel translocation $\mathrm{t}(2 ; 16)$ producing an in-frame fusion of FUS and FEV. J Mol Diagn 9: 459-463, 2007.

3. Urano F, Umezawa A, Yabe H, Hong W, Yoshida K, Fujinaga K and Hata J: Molecular analysis of Ewing's sarcoma: Another fusion gene, EWS-E1AF, available for diagnosis. Jpn J Cancer Res 89: 703-711, 1998.
4. Grier HE, Krailo MD, Tarbell NJ,Link MP, Fryer CJ,Pritchard DJ, Gebhardt MC, Dickman PS, Perlman EJ, Meyers PA, et al: Addition of ifosfamide and etoposide to standard chemotherapy for Ewing's sarcoma and primitive neuroectodermal tumor of bone. N Engl J Med 348: 694-701, 2003.

5. Mora J, Cruz CO, Parareda A and de Torres C: Treatment of relapsed/refractory pediatric sarcomas with gemcitabine and docetaxel. J Pediatr Hematol Oncol 31: 723-729, 2009.

6. Rapkin L, Qayed M, Brill P, Martin M, Clark D, George BA, Olson TA, Wasilewski-Masker K, Alazraki A and Katzenstein HM: Gemcitabine and docetaxel (GEMDOX) for the treatment of relapsed and refractory pediatric sarcomas. Pediatr Blood Cancer 59: 854-858, 2012.

7. Fox E, Patel S, Wathen JK, Schuetze S, Chawla S, Harmon D, Reinke D, Chugh R, Benjamin RS and Helman LJ: Phase II study of sequential gemcitabine followed by docetaxel for recurrent Ewing sarcoma, osteosarcoma, or unresectable or locally recurrent chondrosarcoma: Results of Sarcoma Alliance for Research Through Collaboration Study 003. Oncologist 17: $321,2012$.

8. García-del-Muro X, López-Pousa A, Maurel J, Martín J, Martínez-Trufero J, Casado A, Gómez-España A, Fra J, Cruz J, Poveda A, et al: Randomized phase II study comparing gemcitabine plus dacarbazine versus dacarbazine alone in patients with previously treated soft tissue sarcoma: A Spanish Group for Research on Sarcomas study. J Clin Oncol 29: 2528-2533, 2011.

9. D'Incalci $M$ and Galmarini CM: A review of trabectedin (ET-743): A unique mechanism of action. Mol Cancer Ther 9: 2157-2163, 2010.

10. Garcia-Carbonero R, Supko JG, Manola J, Seiden MV, Harmon D, Ryan DP, Quigley MT, Merriam P, Canniff J, Goss G, et al: Phase II and pharmacokinetic study of ecteinascidin 743 in patients with progressive sarcomas of soft tissues refractory to chemotherapy. J Clin Oncol 22: 1480-1490, 2004.

11. Le Cesne A, Blay JY, Judson I, van Oosterom A, Verweij J, Radford J, Lorigan P, Rodenhuis S, Ray-Coquard I, Bonvalot S, et al: Phase II study of ET-743 in advanced soft tissue sarcomas: A European Organisation for the Research and Treatment of Cancer (EORTC) soft tissue and bone sarcoma group trial. J Clin Oncol 23: 576-584, 2005.

12. Yovine A, Riofrio M, Blay JY, Brain E, Alexandre J, Kahatt C, Taamma A, Jimeno J, Martin C, Salhi Y, et al: Phase II study of ecteinascidin-743 in advanced pretreated soft tissue sarcoma patients. J Clin Oncol 22: 890-899, 2004.

13. Demetri GD, Chawla SP, von Mehren M, Ritch P, Baker LH, Blay JY, Hande KR, Keohan ML, Samuels BL, Schuetze S, et al: Efficacy and safety of trabectedin in patients with advanced or metastatic liposarcoma or leiomyosarcoma after failure of prior anthracyclines and ifosfamide: Results of a randomized phase II study of two different schedules. J Clin Oncol 27: 4188-4196, 2009.

14. Le Cesne A, Cresta S, Maki RG, Blay JY, Verweij J, Poveda A, Casali PG, Balaña C, Schöffski P, Grosso F, et al: A retrospective analysis of antitumor activity with trabectedin in translocation-related sarcomas. Eur J Cancer 48: 3036-3044, 2012.

15. Pink D, Bertz-Lepel J, Busemann C, Bitz U and Reichardt P: Efficacy of trabectedin in patients with advanced or metastatic alveolar soft-part sarcoma. Onkologie 35: 249-252, 2012.

16. Grosso F, Dileo P, Sanfilippo R, Stacchiotti S, Bertulli R, Piovesan C, Jimeno J, D'Incalci M, Gescher A and Casali PG: Steroid premedication markedly reduces liver and bone marrow toxicity of trabectedin in advanced sarcoma. Eur J Cancer 42: 1484-1490, 2006

17. Sanfilippo R, Dileo P, Blay JY, Constantinidou A, Le Cesne A, Benson C, Vizzini L, Contu M, Daldi GG, Dei Tos AP and Casali PG: Trabectedin in advanced synovial sarcomas: A multicenter retrospective study from four European institutions and the Italian Rare Cancer Network. Anticancer Drugs 26: 678-681, 2015.

18. Takahashi S, Araki N, Sugiura H, Ueda T, Takahashi M, Morioka H, Yonemoto T, Hiraga H, Hiruma T, Kunisada T, et al: A randomized phase II study comparing trabectedin $(\mathrm{T})$ and best supportive care (BSC) in patients (pts) with translocationrelated sarcomas (TRS). J Clin Oncol (ASCO Annual Meeting abstracts) 32 (Suppl; abstr 10524): 5s, 2014.

19. Grohar PJ, Griffin LB, Yeung C, Chen QR, Pommier Y, Khanna C, Khan J and Helman LJ: Ecteinascidin 743 interferes with the activity of EWS-FLI1 in Ewing sarcoma cells. Neoplasia 13: 145-153, 2011. 
20. Lau L, Supko JG, Blaney S, Hershon L, Seibel N, Krailo M, Qu W, Malkin D, Jimeno J, Bernstein M, et al: A phase I and pharmacokinetic study of ecteinascidin-743 (Yondelis) in children with refractory solid tumors. A Children's Oncology Group study. Clin Cancer Res 11: 672-677, 2005.

21. Chu Q, Mita A, Forouzesh B, Tolcher AW, Schwartz G, Nieto A Soto-Matos A, Alfaro V, Lebedinsky C and Rowinsky EK: Phase I and pharmacokinetic study of sequential paclitaxe and trabectedin every 2 weeks in patients with advanced solid tumors. Clin Cancer Res 16: 2656-2665, 2010.

22. Dileo P, Grosso F, Casanova M, Jimeno J, Marsoni S, Sanfilippo R, Podda M, Ferrari S, Bertulli R and Casali P: Trabectedin (T) in metastatic Ewing's family tumors (EFT) patients (pts) progressing after standard chemotherapy. Journal of Clinical Oncology, 2007.ASCO Annual Meeting Proceedings (Post-Meeting Edition). Vol 25, No 18S (June 20 Supplement): 10040, 2007.
23. Baruchel S, Pappo A, Krailo M, Baker KS, Wu B, Villaluna D, Lee-Scott M, Adamson PC and Blaney SM: A phase II trial of trabectedin in children with recurrent rhabdomyosarcoma, Ewing sarcoma and non-rhabdomyosarcoma soft tissue sarcomas: A report from the Children's Oncology Group. Eur J Cancer 48: $579-585,2012$.

24. Grohar PJ, Segars LE, Yeung C, Pommier Y, D'Incalci M, Mendoza A and Helman LJ: Dual targeting of EWS-FLI1 activity and the associated DNA damage response with trabectedin and SN38 synergistically inhibits Ewing sarcoma cell growth. Clin Cancer Res 20: 1190-1203, 2014.

25. Cesne AL, Judson I, Maki R, Grosso F, Schuetze S, Mehren MV, Chawla SP, Demetri GD, Nieto A, Tanovic A and Blay JY: Trabectedin is a feasible treatment for soft tissue sarcoma patients regardless of patient age: A retrospective pooled analysis of five phase II trials. Br J Cancer 109: 1717-1724, 2013. 